\title{
Applications des entiers à diviseurs denses
}

\author{
par \\ ERIC SAIAS (Paris)
}

A la mémoire de Paul Erdős

\section{Introduction}

1a. Résultats relatifs aux entiers à diviseurs denses. Désignons par $P^{-}(n)$ le plus petit facteur premier de l'entier $n \geq 2$ et posons

$$
F(n)= \begin{cases}1 & (n=1) \\ \max \left\{d P^{-}(d): d \mid n, d>1\right\} & (n \geq 2)\end{cases}
$$

Nous appelons entiers à diviseurs t-denses les entiers $n$ vérifiant $F(n) \leq n t$. Nous avons choisi cette dénomination en raison de l'identité

$$
\frac{F(n)}{n}=\max _{1 \leq i<\tau(n)} \frac{d_{i+1}(n)}{d_{i}(n)} \quad(n \geq 2),
$$

où $1=d_{1}(n)<\ldots<d_{\tau(n)}=n$ désigne la suite croissante de tous les diviseurs de $n$ (voir le Lemme 2.2 de [9]).

L'objet du présent travail est d'étudier deux problèmes distincts, le premier relatif au petit crible d'Erdős et Ruzsa et le second au graphe divisoriel. Pour cela, nous sommes amenés à utiliser les ordres de grandeur exacts de fonctions de répartition liées aux entiers à diviseurs denses, que nous allons donner maintenant.

Posons

$$
A(x)=\operatorname{card}\{n: F(n) \leq x\} .
$$

Les premiers à étudier cette fonction ont été Schinzel et Szekeres qui ont montré dans [8] que l'on a

$$
A(x)=o(x) \quad \text { quand } x \text { tend vers }+\infty .
$$

Ce résultat a été depuis précisé dans un premier temps par Ruzsa [4] qui a établi l'existence d'une constante $c>0$ pour laquelle

1991 Mathematics Subject Classification: 11N25, 05C38, $20 B 99$. 


$$
A(x) \leq \frac{x}{\log ^{c} x},
$$

puis par Tenenbaum dont les travaux [9] et [10] montrent que pour $\varepsilon>0$,

$$
\frac{x}{(\log x)(\log \log x)^{5 / 3+\varepsilon}} \ll_{\varepsilon} A(x) \ll \frac{x}{\log x} \log \log x .
$$

Enfin il résulte facilement de notre travail [7] que l'on a en fait $\left({ }^{1}\right)$

$$
A(x) \asymp \frac{x}{\log x} .
$$

Pour les applications, nous sommes amenés à considérer des fonctions de répartition plus générales. Posons

$$
\begin{aligned}
D(x, t) & =\operatorname{card}\{n \leq x: F(n) \leq n t\} \\
D^{\prime}(x, t) & =\operatorname{card}\{n \leq x: F(n) \leq n t \text { et } n \text { est sans facteur carré }\}
\end{aligned}
$$

et

$$
A(x, t)=\operatorname{card} \mathcal{A}(x, t) \quad \text { avec } \quad \mathcal{A}(x, t)=\{n \leq x: F(n) \leq x t\} .
$$

Posons de plus

$$
B(x)=\operatorname{card} \mathcal{B}(x)
$$

avec

$$
\mathcal{B}(x)=\{n \leq x: n \notin \mathcal{A}(x, 1) \text { et }[(d \mid n \text { et } d<n) \Rightarrow d \in \mathcal{A}(x, 1)]\} .
$$

Il résulte facilement des résultats de [7] que l'on a $\left({ }^{2}\right)$

$$
D^{\prime}(x, 2 t) \asymp D(x, 2 t) \asymp A(x, t) \asymp x \frac{\log 2 t}{\log 2 x} \quad \text { pour } x \geq t \geq 1 .
$$

Par ailleurs, nous montrons au Lemme 6 du présent travail que l'on a

$$
B(x) \asymp \frac{x}{\log x} .
$$

1b. Petit crible d'Erdös et Ruzsa. Soit $\mathcal{E}$ un ensemble d'entiers strictement positifs. On désigne par $F(x, \mathcal{E})$ le nombre d'entiers strictement positifs $\leq x$ qui n'ont aucun diviseur dans $\mathcal{E}$. Nous nous intéressons ici au problème soulevé par Erdős et Ruzsa de l'estimation asymptotique de la quantité

$$
H(x)=\min F(x, \mathcal{E})
$$

$\left({ }^{1}\right)$ Pour une preuve complète de cette estimation, voir la note $\left({ }^{2}\right)$ où la formule plus générale (2) est établie.

$\left({ }^{2}\right)$ Les estimations de $A(x, t)$ pour $x \geq t \geq 2$ et de $D(x, 2 t)$ et $D^{\prime}(x, 2 t)$ pour $x \geq$ $2 t \geq 2$ résultent directement du Théorème 1 de [7]. De plus, quand $x \geq 4$ et $1 \leq t<2$, on estime $A(x, t)$ en se ramenant au cas général par l'intermédiaire de l'encadrement $A(x / 2,2 t) \leq A(x, t) \leq A(x, 2 t)$. Par ailleurs, quand $x \geq 2$ et $1 \leq t \leq x<2 t$, on estime $D(x, t)$ et $D^{\prime}(x, t)$ en se ramenant au cas général par l'intermédiaire des formules $D(x, 2 t)=D(x, x)$ et $D^{\prime}(x, 2 t)=D^{\prime}(x, x)$. Enfin, les estimations annoncées sont triviales quand $x$ reste borné. 
où le minimum porte sur les ensembles $\mathcal{E}$ vérifiant les conditions

$$
\sum_{e \in \mathcal{E}} \frac{1}{e} \leq 1 \quad \text { et } \quad 1 \notin \mathcal{E}
$$

Dans [4], Ruzsa montre comment déduire de manière élémentaire du théorème des nombres premiers la minoration

$$
H(x) \gg x / \log x .
$$

Le travail de Ruzsa permet également de majorer $H(x)$ en fonction de $A(x)$ par l'intermédiaire de la formule suivante $\left({ }^{3}\right)$ :

$$
H(x) \leq A(x)(1+3 \log (2 x / A(x)))+\sqrt{x} .
$$

Ruzsa conjecture de plus que l'on peut améliorer un certain lemme (voir dans [4] le Lemme 2.8 et la conjecture juste avant le Lemme 2.10) de telle manière à obtenir $H(x) \ll A(x)$ au lieu de (5), ce qui établirait avec (1) et (4) que

$$
H(x) \asymp \frac{x}{\log x} .
$$

Nous ne savons pas démontrer ou infirmer la conjecture de Ruzsa. Cependant, nous montrons ici que l'on a bien (6). En effet, à l'aide d'une variante de l'argumentation qui mène à (5), on obtient (voir le Lemme 10)

$$
H(x) \leq \max (A(x), B(x)+\sqrt{x})
$$

les estimations (1), (3) et (4) permettant alors de conclure. On a donc le

ThÉORÈme 1 . Il existe deux constantes strictement positives $c_{1}$ et $c_{2}$ telles que pour $x \geq 2$, on ait

$$
c_{1} \frac{x}{\log x} \leq H(x) \leq c_{2} \frac{x}{\log x} .
$$

Nous concluons ce chapitre par deux remarques.

Remarque 1. Signalons qu'il résulte facilement du présent travail que

$$
\sum_{b \in \mathcal{B}(x)} \frac{1}{b}=1+O\left(\frac{1}{\log x}\right) .
$$

Cela étant, on ne sait pas si pour tout $x \geq 2$ on a $\sum_{b \in \mathcal{B}(x)} 1 / b<1$, comme semble l'indiquer les calculs effectifs des ensembles $\mathcal{B}(x)$ pour les petits $x$. Il est à noter qu'en cas de réponse positive à cette dernière question, la

$\left({ }^{3}\right)$ Montrons la formule (5). Si $\sum_{b \in \mathcal{B}(x)} 1 / b \leq 1$, on a directement $H(x) \leq F(x, \mathcal{B}(x))$ $=A(x)$. Sinon on note $\mathcal{B}^{\prime}(x)$ une partie maximale de $\mathcal{B}(x)$ telle que $\sum_{b \in \mathcal{B}^{\prime}(x)} 1 / b \leq 1$. On a alors $H(x) \leq F\left(x, \mathcal{B}^{\prime}(x)\right) \leq F(x, \mathcal{B}(x))+x \sum_{b \in \mathcal{B}(x) \backslash \mathcal{B}^{\prime}(x)} 1 / b=A(x)+S-S^{\prime}$ avec $S:=x \sum_{b \in \mathcal{B}(x)} 1 / b \leq x+3 A(x) \log (2 x / A(x))$ d'après le Lemme 7.1 de [9], et $S^{\prime}:=$ $x \sum_{b \in \mathcal{B}^{\prime}(x)} 1 / b>x-\sqrt{x}$ car tous les entiers de $\mathcal{B}(x)$ sont $>\sqrt{x}$. 
démonstration de la majoration de $H(x)$ du Théorème 1 s'obtiendrait alors encore plus simplement en écrivant

$$
H(x) \leq F(x, \mathcal{B}(x))=A(x) \asymp x / \log x .
$$

Remarque 2. On s'intéresse ici au problème d'obtenir rapidement une minoration de $A(x)$. En interprétant la formule (5) comme une minoration de $A(x)$ en fonction de $H(x)$, on obtient en combinant (5) et (4) la formule

$$
A(x) \gg \frac{x}{(\log x) \log \log x},
$$

qui est de qualité légèrement inférieure à ce que l'on a obtenu dans [7] (voir la formule (1) ci-dessus). Cependant, il est intéressant de noter que l'on obtient ainsi une minoration de $A(x)$ qui à un facteur $\log \log x$ près est optimale, et cela différemment et plus rapidement que dans [10] ou [7].

1c. Etude du graphe divisoriel. Soit $f(x)$ le nombre maximum d'entiers strictement positifs $n_{1}, \ldots, n_{f(x)}$ deux à deux distincts, inférieurs à $x$ et tels que pour tout $i, n_{i}$ divise $n_{i+1}$ ou $n_{i+1}$ divise $n_{i}$. Soit $g(x)$ le nombre maximum d'entiers strictement positifs $n_{1}, \ldots, n_{g(x)}$ deux à deux distincts, inférieurs à $x$ et vérifiant $\left[n_{i}, n_{i+1}\right] \leq x$ pour tout $i$. On note que l'on a trivialement $f(x) \leq g(x)$.

Le premier résultat concernant la fonction $g(x)$ est dû à Erdős, Freud et Hegyvári. Dans [1] (Theorem 3 et démonstration du Theorem 4), ils ont démontré implicitement que l'on a pour une certaine constante $c>0$,

$$
\frac{x}{\exp (c \sqrt{\log x} \log \log x)} \leq g(x) \leq(1-\log 2+o(1)) x \quad(x \rightarrow+\infty) .
$$

En répondant ainsi à une question de Hegyvári, Pomerance [3] a amélioré la majoration en

$$
g(x)=o(x) \quad(x \rightarrow+\infty) .
$$

Pour sa part, Pollington [2] a établi la minoration

$$
f(x) \geq \frac{x}{\exp ((2+o(1)) \sqrt{(\log x) \log \log x})} \quad(x \rightarrow+\infty) .
$$

Dans [10], Tenenbaum éclaire d'un jour nouveau le problème de l'estimation asymptotique des fonctions $f(x)$ et $g(x)$ en montrant le lien qui existe entre celui-ci et la répartition des entiers à diviseurs denses. Plus précisément, il montre que pour $x$ assez grand, on a

$$
D^{\prime}(x / 4,2) \leq f(x) \leq g(x) \leq 2 D\left(x, \log ^{5} x\right) .
$$

En utilisant (2), on en déduit que

$$
\frac{x}{\log x} \ll f(x) \leq g(x) \ll \frac{x}{\log x} \log \log x .
$$


Dans [5], nous nous sommes intéressés à la variante suivante de la fonction $f(x)$. Soit $f^{*}(x)$ le nombre maximum d'entiers deux à deux distincts de l'intervalle $[\sqrt{x}, x], n_{1}, \ldots, n_{f^{*}(x)}$, tels que pour tout $i, n_{i}$ divise $n_{i+1}$ ou $n_{i+1}$ divise $n_{i}$. (Notons que l'on a trivialement $f(x) \geq f^{*}(x)$.) En combinant le résultat démontré à la Proposition de [5] et la formule (2) du présent travail, on obtient

$$
f^{*}(x) \gg \frac{x}{\log x} .
$$

Nous montrons ici que l'ordre de grandeur exact des trois fonctions $f^{*}(x)$, $f(x)$ et $g(x)$ est $x / \log x$.

THÉORÈME 2. Il existe deux constantes strictement positives $c_{3}$ et $c_{4}$ telles que pour $x \geq 2$, on ait

$$
c_{3} \frac{x}{\log x} \leq f^{*}(x) \leq f(x) \leq g(x) \leq c_{4} \frac{x}{\log x} .
$$

Donnons la démarche générale des démonstrations des majorations de (7) et $(9)$. Désignons par $\left(n_{i}\right)$ une suite d'entiers $\leq x$, deux à deux distincts et vérifiant $\left[n_{i}, n_{i+1}\right] \leq x$. Dans [10], Tenenbaum montre que si $t$ est suffisamment grand, il y a très peu d'entiers $n_{i}$ qui n'appartiennent pas à $\mathcal{A}(x, t)$. De manière quantitative, on obtient rapidement en suivant sa méthode (cf. Lemme 12)

$$
\operatorname{card}\left\{i: n_{i} \notin \mathcal{A}(x, t)\right\} \ll x(\log x) / t,
$$

d'où, en choisissant $t=\log ^{2} x$,

$$
g(x) \ll A\left(x, \log ^{2} x\right)+x / \log x,
$$

ce qui est essentiellement équivalent à la majoration de (7).

Notre contribution est ici de montrer que par une analyse plus approfondie de la structure des suites $\left(n_{i}\right)$, on obtient

$$
\operatorname{card}\left\{i: n_{i} \notin \mathcal{A}(x, 1)\right\} \ll x / \log x,
$$

d'où

$$
g(x) \ll A(x, 1)+x / \log x \asymp x / \log x .
$$

La motivation initiale d'Erdős, Freud et Hegyvári [1] est l'étude du comportement asymptotique du p.p.c.m. de $a_{i}$ et $a_{i+1}$ où $\left(a_{i}\right)_{i \geq 1}$ est une permutation de $\mathbb{N}^{*}$. En effet, les deux problèmes sont liés par l'intermédiaire de la formule

$$
g\left(\max _{1 \leq i \leq j}\left[a_{i}, a_{i+1}\right]\right) \geq j+1 .
$$

La majoration de $g(x)$ du Théorème 2 nous permet donc d'en déduire le résultat suivant, qui précise la minoration d'Erdős, Freud et Hegyvári correspondante ([1], Theorem 4$)$. 
ThÉORÈme 3. Pour toute permutation $\left(a_{j}\right)_{j \geq 1}$ de $\mathbb{N}^{*}$, on a

$$
\limsup _{j \rightarrow+\infty} \frac{\left[a_{j}, a_{j+1}\right]}{j \log j}>0 \text {. }
$$

2. Quelques notations. Les réels $x, y, z$ et $t$ étant donnés, on notera

$$
u=\frac{\log x}{\log y}, \quad v=\frac{\log x}{\log z} \quad \text { et } \quad w=\frac{\log x}{\log t} .
$$

On désigne par $p$ ou $q$ un nombre premier générique.

On désigne respectivement par $(m, n)$ et $[m, n]$ le p.g.c.d. et le p.p.c.m. de $m$ et $n$. On désigne par $P^{-}(n)$ (resp. $\left.P^{+}(n)\right)$ le plus petit (resp. grand) facteur premier de l'entier $n \geq 2$. On pose de plus $P^{-}(1)=+\infty$ et $P^{+}(1)=$ 1. On désigne par $P^{+}(n)=p_{1}(n) \geq \ldots \geq p_{\Omega(n)}(n)=P^{-}(n)$ la suite décroissante des facteurs premiers de $n$. On rappelle que l'on note

$$
F(n)= \begin{cases}1 & (n=1), \\ \max \left\{d P^{-}(d): d \mid n, d>1\right\} & (n \geq 2) .\end{cases}
$$

On note

$$
\Psi(x, y)=\operatorname{card}\left\{n \leq x: P^{+}(n) \leq y\right\}
$$

et

$$
\Theta(x, y, z)=\operatorname{card}\left\{n \leq x: P^{+}(n) \leq y \text { et } P^{-}(n)>z\right\} .
$$

On note

$$
\begin{aligned}
\mathcal{A}(x, y, z, t) & =\left\{n \leq x: P^{+}(n) \leq y, P^{-}(n)>z \text { et } F(n) \leq x t\right\}, \\
\mathcal{A}(x, z, t) & =\mathcal{A}(x, x, z, t), \\
\mathcal{A}(x, t) & =\mathcal{A}(x, x, 1, t) \quad \text { et } \quad \mathcal{A}(x)=\mathcal{A}(x, x, 1,1)
\end{aligned}
$$

avec les cardinaux

$$
\begin{aligned}
A(x, y, z, t) & =\operatorname{card} \mathcal{A}(x, y, z, t), \quad A(x, z, t)=\operatorname{card} \mathcal{A}(x, z, t), \\
A(x, t) & =\operatorname{card} \mathcal{A}(x, t) \quad \text { et } \quad A(x)=\operatorname{card} \mathcal{A}(x) .
\end{aligned}
$$

On note également

$$
\mathcal{B}(x, t)=\{n \leq x: n \notin \mathcal{A}(x, t) \text { et }[(d \mid n \text { et } d<n) \Rightarrow d \in \mathcal{A}(x, t)]\}
$$

et

$$
\mathcal{B}(x)=\mathcal{B}(x, 1)
$$

avec les cardinaux

$$
B(x, t)=\operatorname{card} \mathcal{B}(x, t) \quad \text { et } \quad B(x)=\operatorname{card} \mathcal{B}(x) .
$$

On note encore

$$
\begin{aligned}
D(x, y, z, t) & =\operatorname{card}\left\{n \leq x: P^{-}(n)>z, P^{+}(n) \leq y \text { et } F(n) \leq n t\right\}, \\
D(x, t) & =\operatorname{card}\{n \leq x: F(n) \leq n t\}
\end{aligned}
$$


et

$$
D^{\prime}(x, t)=\operatorname{card}\{n \leq x: F(n) \leq n t \text { et } n \text { sans facteur carré }\} .
$$

On pose enfin $\widetilde{\varrho}(u)=\varrho(\max (u, 0))$ où $\varrho$ désigne la fonction de Dickman (voir par exemple [7], §4) et

$$
\widetilde{A}(x, y, z, t)=\left\{\begin{array}{cc}
\frac{x}{w \log z}\left(1-\frac{1}{\log _{2}(\max (w, 16))}\right) \widetilde{\varrho}\left(u\left(1-\frac{1}{\sqrt{\log y}}\right)-1\right) \\
\left(0<u<3 \log ^{1 / 3} x\right), \\
\Theta(x, y, z) & \left(u \geq 3 \log ^{1 / 3} x\right) .
\end{array}\right.
$$

3. Majoration de $A(x, y, z, t)$. Notre objectif est ici d'établir la majoration de $A(x, z, t)$ du Lemme 5 , ce qui nous permettra d'estimer au paragraphe suivant certaines quantités dépendant de la répartition des entiers de $\mathcal{A}(x)$ (voir les Lemmes 6 et 7). Comme dans [7], nous allons en fait majorer la quantité plus générale $A(x, y, z, t)$.

Pour cela, l'idée consiste à reprendre la preuve de la majoration de $A(x, y, 1, t)$ faite dans [7] en effectuant quelques modifications mineures. En particulier dans [7], on a commencé par majorer $D(x, y, 1, t)$, puis utilisé un argument élémentaire pour en déduire une majoration de $A(x, y, 1, t)$. En fait, ce détour est inutile comme nous allons le montrer ici en majorant directement $A(x, y, z, t)$.

Techniquement, c'est le Lemme 1 ci-dessous qui va nous permettre de reprendre, pour majorer $A(x, y, z, t)$, la méthode qui nous a permis de majorer $A(x, y, 1, t)$ dans [7].

Lemme 1. Pour $x^{3} \geq z \geq 3 / 2$ et $y \geq 2$, on $a$

$$
\Theta(x, y, z) \ll \frac{\Psi(x, y)}{\log z} .
$$

Démonstration. Cela résulte facilement du Théorème 5 de [6].

Les deux résultats suivants constituent les deux lemmes clefs de la procédure de majoration. (On rappelle pour le Lemme 3 que l'on note $u=$ $(\log x) / \log y$ et $w=(\log x) / \log t$.

Lemme 2. Pour $x \geq 1, y \geq 1, z \geq 1$ et $t \geq 1$, on a

$$
A(x, y, z, t)=1+\sum_{z<p \leq \min (y, \sqrt{x t})} A(x / p, p, z, t) .
$$

LEMME 3. Il existe une constante $w_{0} \geq 16$ telle que sous les conditions $x \geq z \geq 3 / 2, y \geq 2, t \geq 2, w \geq w_{0}$ et $0<u<3 \log ^{1 / 3} x$, on ait

$$
\widetilde{A}(x, y, z, t) \geq 1+\sum_{z<p \leq \min (y, \sqrt{x t})} \widetilde{A}(x / p, p, z, t) .
$$


On obtient la formule du Lemme 2 en classant les entiers comptés dans $A(x, y, z, t)$ suivant leur plus grand facteur premier.

Démonstration (du Lemme 3). Désignons par $\mu(x)$ le réel défini pour $x$ suffisamment grand par

$$
\log ^{2 / 3}(x / \mu(x))=3 \log \mu(x) \quad \text { et } \quad 2 \leq \mu(x) \leq x .
$$

En utilisant le Lemme 1 pour la seconde inégalité, pour une constante $c$ convenable on a

$$
\begin{aligned}
& 1+\sum_{z<p \leq \min (y, \sqrt{x t})} \widetilde{A}(x / p, p, z, t) \\
& \leq \Theta(x, \mu(x), z)+\sum_{\mu(x)<p \leq \min (y, \sqrt{x t})} \widetilde{A}(x / p, p, z, t) \\
& \leq \frac{c \Psi(x, \mu(x))}{\log z}+\sum_{\mu(x)<p \leq \min (y, \sqrt{x t})} \widetilde{A}(x / p, p, z, t) .
\end{aligned}
$$

Il suffit alors de reprendre la preuve du Lemme 9 de [7] pour conclure.

Lemme 4. Sous les conditions $x^{3} \geq z \geq 3 / 2, y \geq 2$ et $t \geq 2$, on a

$$
A(x, y, z, t) \ll \widetilde{A}(x, y, z, t) .
$$

La méthode de démonstration est identique à celle de la Proposition 1 de [7]. Elle consiste dans un premier temps à établir de manière directe la majoration demandée dans un certain domaine en $x, y, z, t$, puis à établir cette majoration dans le domaine complémentaire à l'aide d'une récurrence qui utilise les Lemmes 2 et 3 . Voyons cela.

Démonstration (du Lemme 4). Soit $w_{0}$ une constante convenable pour le Lemme 3 . On suppose que $x, y, z$ et $t$ vérifient les hypothèses du lemme. On distingue plusieurs cas.

$1^{\text {er }}$ cas : $u \geq 3 \log ^{1 / 3} x$. On a alors $A(x, y, z, t) \leq \Theta(x, y, z)=$ $\widetilde{A}(x, y, z, t)$.

$2^{e}$ cas $: 0<u<3 \log ^{1 / 3} x$ et $x^{3} \geq z>x$. On a alors $A(x, y, z, t)=1 \ll$ $\widetilde{A}(x, y, z, t)$.

$3^{e}$ cas : $0<u<3 \log ^{1 / 3} x$ et $w<w_{0}$. On sait alors (voir les Lemmes 3 et 2 (iv) de [7]) que $\Psi(x, y) \ll x \varrho(u) \ll x \widetilde{\varrho}(u-1) /(u+1)$. D'où avec le Lemme 1,

$$
\begin{aligned}
A(x, y, z, t) & \leq \Theta(x, y, z) \ll \frac{\Psi(x, y)}{\log z} \ll \frac{x \widetilde{\varrho}(u-1)}{(u+1) \log z} \\
& \ll \frac{x \widetilde{\varrho}(u-1)}{w \log z} \ll \widetilde{A}(x, y, z, t) .
\end{aligned}
$$

Cela achève la première étape de la preuve. 
Soit $c \geq 1$ une constante pour laquelle on ait

$$
A(x, y, z, t) \leq c \widetilde{A}(x, y, z, t)
$$

dans chacun des trois cas étudiés. Montrons maintenant par récurrence sur $k \geq 0$ que l'on a l'inégalité (10) sous l'hypothèse

$$
x^{3} \geq z \geq 3 / 2, \quad y \geq 2, \quad t \geq 2 \quad \text { et } \quad x \leq 2^{k} .
$$

D'après le travail fait ci-dessus, on peut omettre l'étape d'initialisation de la récurrence et supposer que l'on a

$$
x \geq z, \quad w \geq w_{0} \quad \text { et } \quad 0<u<3 \log ^{1 / 3} x .
$$

On suppose également, d'une part que sous la condition $\left(\mathrm{H}_{k}\right)$ on a (10), et d'autre part que l'hypothèse $\left(\mathrm{H}_{k+1}\right)$ est vérifiée. Alors, l'inégalité $x \leq 2^{k+1}$ entraîne que pour tout nombre premier $p$, on a $x / p \leq 2^{k}$. De plus, comme $w \geq w_{0} \geq 16>3$ et $x \geq z$, l'inégalité $p \leq \sqrt{x t}$ entraîne $(x / p)^{3} \geq z$. Donc les quadruplets $(x / p, p, z, t)$ tels que $z<p \leq \min (y, \sqrt{x t})$ vérifient la condition $\left(\mathrm{H}_{k}\right)$. En utilisant successivement le Lemme 2, l'hypothèse de récurrence appliquée aux $(x / p, p, z, t)$ et le Lemme 3 , on obtient

$$
\begin{aligned}
A(x, y, z, t) & =1+\sum_{z<p \leq \min (y, \sqrt{x t})} A(x / p, p, z, t) \\
& \leq c\left(1+\sum_{z<p \leq \min (y, \sqrt{x t})} \widetilde{A}(x / p, p, z, t)\right) \leq c \widetilde{A}(x, y, z, t) .
\end{aligned}
$$

Cela achève la preuve du Lemme 4 .

Lemme 5. Sous les conditions $x \geq z \geq 1$ et $t \geq 1$, on a

$$
A(x, z, t) \ll \frac{x \log (2 \min (x, t))}{(\log 2 x) \log 2 z} .
$$

Démonstration. On a $A(x, z, t) \leq A(2 x, 2 x, \max (z, 3 / 2), 2 t)$. On conclut en appliquant le Lemme 4 à cette dernière quantité.

4. Etude des ensembles $\mathcal{A}(x, t)$ et $\mathcal{B}(x, t)$. Rappelons que l'on note $p_{1}(n) \geq p_{2}(n) \geq \ldots \geq p_{\Omega(n)}(n)$ la suite décroissante des facteurs premiers de $n$ de telle sorte que $n=\prod_{k=1}^{\Omega(n)} p_{k}(n)$. Il est facile de vérifier que l'on a

$$
\mathcal{A}(x, t)=\{1\} \cup\left\{2 \leq n \leq x: \max _{1 \leq k \leq \Omega(n)} p_{1}(n) \ldots p_{k-1}(n) p_{k}(n)^{2} \leq x t\right\}
$$

et

$$
\begin{aligned}
\mathcal{B}(x, t)=\left\{2 \leq n \leq x: \max _{1 \leq k<\Omega(n)} p_{1}(n)\right. & \ldots p_{k-1}(n) p_{k}(n)^{2} \leq x t \\
& \left.<p_{1}(n) \ldots p_{\Omega(n)-1}(n) p_{\Omega(n)}(n)^{2}\right\} .
\end{aligned}
$$

C'est sous cette forme que nous allons dorénavant considérer ces ensembles. 
Lemme 6. Pour $x \geq 2$, on a

$$
B(x) \asymp x / \log x .
$$

Démonstration. Les nombres premiers $p$ vérifiant $\sqrt{x}<p \leq x$ sont éléments de $\mathcal{B}(x)$. Donc en utilisant le postulat de Bertrand pour les $x$ petits et le théorème des nombres premiers pour les $x$ grands, on a

$$
B(x) \geq \pi(x)-\pi(\sqrt{x}) \asymp x / \log x .
$$

En écrivant de manière unique tout élément $b$ de $\mathcal{B}(x)$ sous la forme $b=a p$ avec $a \in \mathcal{A}(x)$ et $\sqrt{x / a}<p \leq P^{-}(a)$ et en notant $a=q a^{\prime}$ avec $q=P^{-}(a)$, on obtient

$$
\begin{aligned}
B(x) & =\sum_{a \in \mathcal{A}(x)} \sum_{\sqrt{x / a}<p \leq \min \left(P^{-}(a), x / a\right)} 1 \\
& =\pi(x)-\pi(\sqrt{x})+\sum_{\substack{q \leq \sqrt{x} \\
a^{\prime} \in \mathcal{A}(x) \\
a^{\prime} \leq x / q^{2} \\
q \leq P^{-}\left(a^{\prime}\right)}} \sum_{\sqrt{x /\left(a^{\prime} q\right)}<p \leq q} 1 \\
& \leq \pi(x)+\sum_{q \leq \sqrt{x}} A\left(x / q^{2}, q-0, q^{2}\right) \pi(q) \\
& \ll \pi(x)+x \sum_{q \leq \sqrt{x}} \frac{1}{q(\log q) \log \left(2 x / q^{2}\right)}
\end{aligned}
$$

d'après le Lemme 5 et le théorème des nombres premiers. On montre que la dernière expression est $\ll x / \log x$ en effectuant une sommation d'Abel et en réutilisant le théorème des nombres premiers.

Lemme 7. On pose

$$
\begin{aligned}
Q_{1}(x) & =\sum_{a \in \mathcal{A}(x)} \frac{1}{a \log ^{3}(x / a)}, \\
Q_{2}(x) & =\sum_{\substack{a \in \mathcal{A}(x) \\
a P^{-}(a)^{2}>x}} \frac{1}{a \log ^{3 / 2}(x / a)},
\end{aligned}
$$

et

$$
Q_{3}(x)=\sum_{\substack{a \in \mathcal{A}(x) \\ a P^{-}(a)^{2} \leq x}} \frac{P^{-}(a)^{2}}{\sqrt{\log \left(P^{-}(a)\right)}} .
$$

On a alors pour $x \geq 2$,

$$
Q_{1}(x) \ll \frac{1}{\log x}, \quad Q_{2}(x) \ll \frac{1}{\log x} \quad \text { et } \quad Q_{3}(x) \ll \frac{x}{\log x} .
$$


Démonstration. Les calculs sont standards. Pour $Q_{1}(x)$, on effectue une sommation d'Abel qui permet de se ramener à la majoration d'intégrales dépendant de $A(x, z, t)$. Le Lemme 5 permet alors de se ramener à la majoration d'intégrales banales. Pour les sommes $Q_{2}(x)$ et $Q_{3}(x)$, on commence par classer les entiers $a$ suivant leur plus petit facteur premier $q$ comme dans la démonstration du Lemme 6 . On obtient alors une somme double en $q$ et en $a^{\prime}$ avec $a^{\prime}=a / q$. On effectue une sommation d'Abel pour transformer la somme en $a^{\prime}$ en une intégrale dépendant de $A(x, z, t)$. On majore par le Lemme 5 et on effectue une nouvelle sommation d'Abel pour transformer la somme en $q$ en une intégrale dépendant de $\pi(x)$. Le théorème des nombres premiers permet alors de se ramener à la majoration d'intégrales banales. Nous laissons au lecteur le soin de vérifier que l'on obtient bien ainsi les majorations annoncées.

Lemme 8. Soient $x$ et $t$ deux réels tels que $x \geq 2$ et $1 \leq t<x$. Soit $n$ un entier $\leq x$ et n'appartenant pas à $\mathcal{A}(x, t)$. Alors, il existe un unique diviseur $b$ de $n$ qui soit élément de $\mathcal{B}(x, t)$.

Démonstration. Soit $n \leq x, n \notin \mathcal{A}(x, t)$. Il existe un entier $i \leq \Omega(n)$ tel que $p_{1}(n) \ldots p_{i-1}(n) p_{i}(n)^{2}>x t$. Soit $i_{0}$ le plus petit de ces entiers. On obtient un entier $b$ convenable en choisissant $b=p_{1}(n) \ldots p_{i_{0}}(n)$. Pour démontrer le caractère unique de cet entier $b$, il suffit de prouver que les diviseurs $d$ de $n$, distincts de $b$, n'appartiennent pas à $\mathcal{B}(x, t)$. Il est clair que les diviseurs de $n$ qui sont multiple ou diviseur propre de $b$ n'appartiennent pas à $\mathcal{B}(x, t)$. Nous allons montrer que dans les autres cas de figure, le diviseur $d$ de $n$ appartient à $\mathcal{A}(x, t)$, et donc pas à $\mathcal{B}(x, t)$. Posons $n=b m$ et

$$
\frac{d}{(d, b)}=q_{1} \ldots q_{k} \quad \text { avec } q_{1} \geq \ldots \geq q_{k} .
$$

On a $q_{1} \leq q_{1} \ldots q_{k} \leq m \leq x / b<P^{-}(b)$. Pour montrer que $d \in \mathcal{A}(x, t)$, il suffit donc de vérifier que $(d, b) \in \mathcal{A}(x, t)$ et que

$$
\text { pour tout } i, 1 \leq i \leq k, \quad(d, b) q_{1} \ldots q_{i-1} q_{i}^{2} \leq x .
$$

La première assertion résulte du fait qu'ici, $(d, b)$ est un diviseur propre de $b$. Pour la seconde, on a

$$
(d, b) q_{1} \ldots q_{i-1} q_{i}^{2} \leq b q_{1} \ldots q_{i-1} q_{i} \leq \frac{b d}{(b, d)}=[b, d] \leq n \leq x,
$$

ce qui conclut.

Le Lemme 2.1 de [4] correspond au cas particulier où $t=1 \mathrm{du}$ lemme suivant.

Lemme 9. Soient $x$ et $t$ deux réels tels que $x \geq 2$ et $1 \leq t<x$. Pour toute paire $\left\{b_{1}, b_{2}\right\}$ d'éléments distincts de $\mathcal{B}(x, t)$, on a $\left[b_{1}, b_{2}\right]>x t$. 
Démonstration. Les deux entiers $b_{1}$ et $b_{2}$ sont éléments de $\mathcal{B}(x, t)$ donc de $\mathcal{B}(x t, 1)$, et par ailleurs diviseurs de $n=\left[b_{1}, b_{2}\right]$. Donc d'après le Lemme 8 , si $\left[b_{1}, b_{2}\right] \leq x t$ alors $b_{1}=b_{2}$.

5. Démonstration du Théorème 1. Le Théorème 1 résulte de la combinaison de la minoration (4), du résultat suivant appliqué à l'ensemble $\mathcal{E}=\mathcal{B}(x)$ (ce qui est justifié par le Lemme 9) et des majorations $F(x, \mathcal{B}(x))=$ $A(x) \ll x / \log x$ et $B(x) \ll x / \log x$, qui résultent des Lemmes 5 et 6 .

Lemme 10. Soit $x \geq 2$. Pour tout ensemble d'entiers $\mathcal{E}$ de l'intervalle $[\sqrt{x}, x]$ vérifiant

$$
(m \in \mathcal{E}, n \in \mathcal{E} \text { et } m \neq n) \Rightarrow([m, n]>x),
$$

on $a$

$$
H(x) \leq \max (F(x, \mathcal{E}), \operatorname{card} \mathcal{E}+\sqrt{x}) .
$$

Démonstration. Si $\sum_{e \in \mathcal{E}} 1 / e \leq 1$, on a $H(x) \leq F(x, \mathcal{E})$. Sinon, on procède comme dans [4] et [9] en considérant une partie maximale $\mathcal{E}^{\prime}$ de $\mathcal{E}$ vérifiant $\sum_{e \in \mathcal{E}^{\prime}} 1 / e \leq 1$. On a alors

$$
H(x) \leq F\left(x, \mathcal{E}^{\prime}\right) \leq F(x, \mathcal{E})+\sum_{e \in \mathcal{E} \backslash \mathcal{E}^{\prime}}\left[\frac{x}{e}\right] .
$$

Or on a d'une part d'après (11),

$$
F(x, \mathcal{E})=[x]-\sum_{e \in \mathcal{E}}\left[\frac{x}{e}\right] \leq x\left(1-\sum_{e \in \mathcal{E}} \frac{1}{e}\right)+\operatorname{card} \mathcal{E},
$$

et d'autre part,

$$
\sum_{e \in \mathcal{E} \backslash \mathcal{E}^{\prime}}\left[\frac{x}{e}\right] \leq x \sum_{e \in \mathcal{E} \backslash \mathcal{E}^{\prime}} \frac{1}{e} \leq x\left(\sum_{e \in \mathcal{E}} \frac{1}{e}-1\right)+\sqrt{x}
$$

car tous les entiers de $\mathcal{E}$ sont $\geq \sqrt{x}$.

6. Nouvelles notations et résultats préliminaires relatifs aux chaînes. On appellera ici chaîne d'entiers $\leq x$ de longueur $l$ tout $l$-uplet d'entiers strictement positifs deux à deux distincts $\mathcal{C}=\left(n_{i}\right)_{1 \leq i \leq l}$, telle que pour tout $i(1 \leq i<l)$, on a $\left[n_{i}, n_{i+1}\right] \leq x$. On note $l(\mathcal{C})$ la longueur de la chaîne $\mathcal{C}$.

Pour une telle chaine $\mathcal{C}=\left(n_{i}\right)_{1 \leq i \leq l}$, on appellera $t$-composante de $\mathcal{C}$ toute chaîne extraite $\mathcal{O}=\left(n_{i}, n_{i+1}, \ldots, n_{j}\right)$ avec $1 \leq i \leq j \leq l$ vérifiant les trois conditions suivantes :

- $n_{\alpha} \notin \mathcal{A}(x, t)$ pour $i \leq \alpha \leq j$,

- $i=1$ ou $n_{i-1} \in \mathcal{A}(x, t)$,

- $j=l$ ou $n_{j+1} \in \mathcal{A}(x, t)$. 
Si $i \neq 1$, l'entier $n_{i-1}$ sera appelé élément d'entrée de la $t$-composante $\mathcal{O}$. On notera $n_{i-1}=e(\mathcal{O})$.

Lemme 11. Soient $x$ et $t$ deux réels tels que $x \geq 2$ et $1 \leq t<x$. Soient $\mathcal{C}$ une chaîne d'entiers $\leq x$ et $\mathcal{O}$ une t-composante de $\mathcal{C}$. Alors il existe un unique entier $b$ de $\mathcal{B}(x, t)$ qui divise tous les entiers de $\mathcal{O}$. On notera dorénavant $b(\mathcal{O})$ cet entier $b$.

Démonstration. D'après le Lemme 8 , chaque élément d'une $t$-composante donnée admet un unique entier de $\mathcal{B}(x, t)$ comme diviseur, et, d'après le Lemme 9 , tous ces entiers sont égaux.

Pour toute chaîne $\mathcal{C}=\left(n_{i}\right)_{1 \leq i \leq l}$ on notera $\chi_{t}(\mathcal{C})$ l'ensemble de ses $t$-composantes $\left(n_{\alpha}\right)_{i \leq \alpha \leq j}$ telles que $i \neq 1$.

Les deux lemmes suivants constituent une généralisation du travail effectué par Tenenbaum [10] pour majorer $g(x)$.

Lemme 12. Soient $x$ et $t$ deux réels tels que $x \geq 2$ et $1 \leq t \leq x$. Soit $\mathcal{C}$ une chaîne d'entiers $\leq x$. On a

$$
\#\{n \in \mathcal{C}: n \notin \mathcal{A}(x, t)\} \ll \frac{x \log x}{t} .
$$

Démonstration. Pour toute $t$-composante $\mathcal{O}$ de $\chi_{t}(\mathcal{C})$, on a

$$
e(\mathcal{O}) P^{-}(b(\mathcal{O})) \leq[e(\mathcal{O}), b(\mathcal{O})] \leq x .
$$

Donc d'après le Lemme 11,

$$
\begin{aligned}
\sum_{\mathcal{O} \in \chi_{t}(\mathcal{C})} l(\mathcal{O}) & \leq x \sum_{\mathcal{O} \in \chi_{t}(\mathcal{C})} \frac{1}{b(\mathcal{O})} \leq \frac{1}{t} \sum_{\mathcal{O} \in \chi_{t}(\mathcal{C})} P^{-}(b(\mathcal{O})) \\
& \leq \frac{x}{t} \sum_{\mathcal{O} \in \chi_{t}(\mathcal{C})} \frac{1}{e(\mathcal{O})} \ll \frac{x \log x}{t} .
\end{aligned}
$$

Par ailleurs, il y a au plus une $t$-composante de $\mathcal{C}$ qui n'appartient pas à $\chi_{t}(\mathcal{C})$. Si elle existe, notons-la $\mathcal{O}_{0}$. On a alors

$$
l\left(\mathcal{O}_{0}\right) \leq \frac{x}{b\left(\mathcal{O}_{0}\right)}<\sqrt{\frac{x}{t}} \ll \frac{x \log x}{t},
$$

ce qui permet de conclure.

On désignera par $g(x, t)$ le cardinal maximum d'un ensemble $\mathcal{U}=$ $\bigcup_{1 \leq \alpha \leq t} \mathcal{C}_{\alpha}$ où $\left(\mathcal{C}_{\alpha}\right)_{1 \leq \alpha \leq t}$ désigne une famille de $t$ chaînes d'entiers $\leq x$. Notons que $g(x)=g(x, 1)$.

Lemme 13. On a uniformément pour $x \geq 3$ et $\log x \leq t \leq x$,

$$
g(x, t) \ll x \frac{\log t}{\log x} .
$$


Démonstration. C'est clair si $t>x^{1 / 3}$. Supposons donc $t \leq x^{1 / 3}$. Soit $\left(\mathcal{C}_{\alpha}\right)_{1 \leq \alpha \leq t}$ une famille de $t$ chaînes d'entiers $\leq x$ telle que $\# \bigcup_{1 \leq \alpha \leq t} \mathcal{C}_{\alpha}$ $=g(x, t)$. Notons $\mathcal{U}=\bigcup_{1 \leq \alpha \leq t} \mathcal{C}_{\alpha}$. On a

$$
\# \mathcal{U} \cap \mathcal{A}\left(x, t^{3}\right) \leq A\left(x, t^{3}\right) \ll x \frac{\log t}{\log x}
$$

d'après le Lemme 5 . On a aussi d'après le Lemme 12,

$$
\#\left\{n \in \mathcal{U}: n \notin \mathcal{A}\left(x, t^{3}\right)\right\} \ll x(\log x) / t^{2} \leq x / \log x,
$$

ce qui permet de conclure.

Dorénavant, on ne travaillera qu'avec des 1-composantes. Pour simplifier, on écrira donc composante à la place de 1-composante et on notera $\chi(\mathcal{C})$ à la place de $\chi_{1}(\mathcal{C})$.

Nous sommes amenés de plus à donner de nouvelles notations. Pour toute chaîne $\mathcal{C}$ et toute composante $\mathcal{O}$ de $\chi(\mathcal{C})$, on pose

$$
a(\mathcal{O})=(e(\mathcal{O}), b(\mathcal{O})) \quad \text { et } \quad r(\mathcal{O})=b(\mathcal{O}) / a(\mathcal{O})
$$

et pour tout $a$ de $\mathcal{A}(x)$, on note

$$
\chi(\mathcal{C}, a)=\{\mathcal{O} \in \chi(\mathcal{C}): a(\mathcal{O})=a\}, \quad \mathcal{R}(\mathcal{C}, a)=\{r(\mathcal{O}): \mathcal{O} \in \chi(\mathcal{C}, a)\}
$$

et

$$
k(a, r)=\operatorname{card}\{\mathcal{O} \in \chi(\mathcal{C}): a=a(\mathcal{O}) \text { et } r=r(\mathcal{O})\} .
$$

Lemme 14. Soient $\mathcal{C}$ une chaîne d'entiers $\leq x$ et $a \in \mathcal{A}(x)$. Pour tout élément $r$ de $\mathcal{R}(\mathcal{C}, a)$, on a

$$
\max \left(\frac{x}{a P^{-}(a)},\left(\frac{x}{a}\right)^{\Omega(r) /(\Omega(r)+1)}\right)<r \leq \frac{x}{a} .
$$

Démonstration. Pour une certaine composante $\mathcal{O}$ de $\mathcal{C}$, on a $r=$ $b(\mathcal{O}) / a \leq x / a$. Posons par ailleurs $k=\Omega(r)$. On a alors

$$
\begin{aligned}
\min \left(a^{k} r^{k+1},\left(\operatorname{arP} P^{-}(a)\right)^{k}\right) & \geq \min \left(\left(\operatorname{arP} P^{-}(r)\right)^{k},\left(\operatorname{arP} P^{-}(a)\right)^{k}\right) \\
& =\left(\operatorname{arP} P^{-}(a r)\right)^{k}>x^{k} .
\end{aligned}
$$

Lemme 15. Soient $\mathcal{C}$ une chaîne d'entiers $\leq x$ et $a \in \mathcal{A}(x)$. On a

$$
\# \mathcal{R}(\mathcal{C}, a) \leq \# \chi(\mathcal{C}, a)<\min \left(P^{-}(a), \sqrt{x / a}\right) .
$$

Dém o n stration. L'inégalité $\# \mathcal{R}(\mathcal{C}, a) \leq \# \chi(\mathcal{C}, a)$ résulte clairement de la définition de $\mathcal{R}(\mathcal{C}, a)$.

Soit $\mathcal{O}$ une composante de $\chi(\mathcal{C}, a)$. On a

$$
\frac{e(\mathcal{O})}{a}=\frac{[e(\mathcal{O}), b(\mathcal{O})]}{a r(\mathcal{O})} \leq \frac{x}{\operatorname{ar}(\mathcal{O})}<\min \left(P^{-}(a), \sqrt{x / a}\right)
$$

d'après le Lemme 14. Cela permet de conclure car les éléments d'entrée des composantes de $\chi(\mathcal{C}, a)$ sont deux à deux distincts. 
Pour achever ces préliminaires, nous donnons sans démonstration le résultat suivant, qui découle facilement du théorème des nombres premiers.

Lemme 16. Il existe une constante $C$ telle que pour toute partie finie non vide $\mathbb{P}$ de l'ensemble des nombres premiers, on ait

$$
\sum_{p \in \mathbb{P}} \frac{1}{p} \leq C \frac{\log \left(1+\frac{\# \mathbb{P}}{\min \mathbb{P}} \log (\max \mathbb{P})\right)}{\log (\min \mathbb{P})}
$$

7. Démonstration du Théorème 2. D'après (8), pour démontrer le Théorème 2 , il suffit de montrer que $g(x) \ll x / \log x$, ce que nous allons faire maintenant.

Soit $\mathcal{C}=\left(n_{i}\right)_{1 \leq i \leq g(x)}$ une chaîne d'entiers $\leq x$ de longueur $g(x)$. L'ensemble des entiers de $\mathcal{C}$ qui sont éléments de $\mathcal{A}(x)$ sont en nombre $O(x / \log x)$ d'après le Lemme 5. Par ailleurs, si $n_{1}$ est élément d'une composante $\mathcal{O}_{0}$, la longueur de celle-ci, $l\left(\mathcal{O}_{0}\right)$, vérifie

$$
l\left(\mathcal{O}_{0}\right) \leq x / b\left(\mathcal{O}_{0}\right)<\sqrt{x} .
$$

De plus, on a

$$
\operatorname{card} \bigcup_{\mathcal{O} \in \chi(\mathcal{C})} \mathcal{O} \leq \sum_{a \in \mathcal{A}(x)}(S(x, a)+T(x, a))
$$

avec

$$
S(x, a)=\sum_{\substack{r \in \mathcal{R}(\mathcal{C}, a) \\ k(a, r)>\log ^{3}(x / a) \text { ou } r>(x / a)^{2 / 3}}} \frac{x}{a r}
$$

et

$$
T(x, a)=\sum_{\substack{r \in \mathcal{R}(\mathcal{C}, a) \\ r \leq(x / a)^{2 / 3}}} g\left(x / a r, \log ^{3}(x / a)\right) .
$$

Or d'une part, en utilisant les Lemmes 14, 15 et 7, on obtient

$$
\begin{aligned}
& \sum_{a \in \mathcal{A}(x)} S(x, a) \\
& \leq \sum_{a \in \mathcal{A}(x)}\left(\sqrt{x / a} \#\left\{r \in \mathcal{R}(\mathcal{C}, a): k(a, r)>\log ^{3}(x / a)\right\}+(x / a)^{1 / 3} \# \mathcal{R}(\mathcal{C}, a)\right) \\
& \ll \sum_{a \in \mathcal{A}(x)} \frac{\sqrt{x / a}}{\log ^{3}(x / a)} \# \chi(\mathcal{C}, a) \leq x Q_{1}(x) \ll \frac{x}{\log x} .
\end{aligned}
$$

Et d'autre part, en utilisant successivement le Lemme 13, le Lemme 14, le Lemme 16, les Lemmes 14 et 15 et pour finir le Lemme 7, on obtient 


$$
\begin{aligned}
& \frac{1}{x} \sum_{a \in \mathcal{A}(x)} T(x, a) \\
& \ll \sum_{a \in \mathcal{A}(x)} \frac{\log \log (2 x / a)}{a \log (x / a)} \sum_{\substack{r \in \mathcal{R}(\mathcal{C}, a) \\
r \leq(x / a)^{2 / 3}}} \frac{1}{r} \leq \sum_{a \in \mathcal{A}(x)} \frac{\log \log (2 x / a)}{a \log (x / a)} \sum_{p \in \mathcal{R}(\mathcal{C}, a)} \frac{1}{p} \\
& \ll \sum_{a \in \mathcal{A}(x)} \frac{\log \log (2 x / a)}{a \log (x / a) \log (\min \mathcal{R}(\mathcal{C}, a))} \log \left(1+\frac{\# \mathcal{R}(\mathcal{C}, a)}{\min \mathcal{R}(\mathcal{C}, a)} \log (\max \mathcal{R}(\mathcal{C}, a))\right) \\
& \ll \sum_{a \in \mathcal{A}(x)} \frac{\log \log (2 x / a)}{a \log { }^{2}(x / a)} \log \left(1+\min \left(1, \frac{a P^{-}(a)^{2}}{x}\right) \log (x / a)\right) \\
& \ll Q_{2}(x)+\frac{Q_{3}(x)}{x} \ll \frac{1}{\log x} .
\end{aligned}
$$

Cela achève la preuve du Théorème 2 .

\section{Bibliographie}

[1] P. Erdős, R. Freud and N. Hegyvári, Arithmetical properties of permutations of integers, Acta Math. Hungar. 41 (1983), 169-176.

[2] A. D. Pollington, There is a long path in the divisor graph, Ars Combin. 16-B (1983), 303-304.

[3] C. Pomerance, On the longest simple path in the divisor graph, Congr. Numer. 40 (1983), 291-304.

[4] I. Z. Ruzsa, On the small sieve II. Sifting by composite numbers, J. Number Theory 14 (1982), 260-268.

[5] E. Saias, Sur l'utilisation de l'identité de Buchstab, dans : Séminaire de Théorie des Nombres de Paris 1991-92, S. David (ed.), Birkhaüser, 1993, 217-245.

[6] -, Entiers sans grand ni petit facteur premier III, Acta Arith. 71 (1995), 351-379.

[7] -, Entiers à diviseurs denses 1, J. Number Theory 62 (1997), 163-191.

[8] A. Schinzel et G. Szekeres, Sur un problème de M. Paul Erdős, Acta Sci. Math. (Szeged) 20 (1959), 221-229.

[9] G. Tenenbaum, Sur un problème de crible et ses applications, Ann. Sci. Ecole Norm. Sup. (4) 19 (1986), 1-30.

[10] —, Sur un problème de crible et ses applications, 2. Corrigendum et étude du graphe divisoriel, ibid. 28 (1995), 115-127.

Laboratoire de Probabilités

Université Paris VI

4, Pl. Jussieu

75252 Paris Cedex 05, France

E-mail: saias@ccr.jussieu.fr 